\title{
Biologia e exigências térmicas de cinco linhagens de Trichogramma pretiosum Riley (Hymenoptera: Trichogrammatidae) criadas em ovos de Tuta absoluta (Meyrick) (Lepidoptera: Gelechiidae)
}

\author{
Biology and thermal requeriments of five Trichogramma pretiosum Riley (Hymenoptera: \\ Trichogrammatidae) strains, reared on eggs of Tuta absoluta (Meyrick) \\ (Lepidoptera: Gelechiidae)
}

\author{
Dirceu Pratissoli ${ }^{1}$ Edvaldo Fialho dos Reis ${ }^{2}$ Hugo Bolsoni Zago ${ }^{3}$ \\ Patrik Luiz Pastori ${ }^{4}$ Tiago Tamanhoni ${ }^{1}$
}

\section{RESUMO}

\begin{abstract}
$O$ objetivo desta pesquisa foi estudar a biologia e as exigências térmicas de cinco linhagens de Trichogramma pretiosum Riley (Hymenoptera: Trichogrammatidae) coletadas em áreas comerciais de tomate, no Estado do Espírito Santo, em ovos da traça-do-tomateiro Tuta absoluta (Meyrick) (Lepidoptera: Gelechiidae), sob diferentes temperaturas constantes e flutuantes. A duração do ciclo de desenvolvimento (ovo-adulto) das cinco linhagens de T. pretiosum criadas em ovos de T. absoluta foi afetada significativamente pela temperatura, na faixa das constantes $\left(15\right.$ a $\left.30^{\circ} \mathrm{C}\right)$. Esse mesmo fato não foi observado nas temperaturas alternadas $(26 / 14$ e $\left.29 / 16^{\circ} \mathrm{C}\right)$. O desempenho das linhagens nas diferentes temperaturas mostrou que L2 e L5 apresentaram os melhores resultados quanto à taxa de emergência de seus descendentes. A razão sexual das cinco linhagens foi afetada pela temperatura, havendo diferença significativa entre os valores obtidos nas faixas das temperaturas (constante e alternada) estudadas. As linhagens de T. pretiosum apresentaram uma temperatura base (tb) que variou de 8,70 a $13,45^{\circ} \mathrm{C}$, e uma constante térmica (K) de 90,09 a 159,43 graus/dia.
\end{abstract}

Palavras-chave: Insecta, Trichogrammatidae, controle biológico, traça-do-tomateiro.

\section{ABSTRACT}

The objective of this research was to evaluate the biology and the thermal requeriments of five strains of Trichogramma pretiosum Riley (Hymenoptera: Trichogrammatidae) collected in commercial areas of tomato, in Espírito Santo State, on eggs the tomato leafminer Tuta absoluta (Meyrick) (Lepidoptera: Gelechiidae) under constant and alternating temperatures. The duration of the development cycle (egg-adult) of the five strains of $\boldsymbol{T}$. pretiosum reared in eggs of T. absoluta was affected significantly by the temperature, in the band of the constants $\left(15\right.$ to $\left.30^{\circ} \mathrm{C}\right)$. This same fact was not observed in the alternated temperatures (26/14 and 29/ $\left.16^{\circ} \mathrm{C}\right)$. The performance of the strains in the different temperatures, showed that $L 2$ and $L 5$ presented the best results concerning the emergency rate of their descendants of viability. The sex ratio of the five strains was affected by the temperature, with significant differences obtained in all the bands of the temperatures (constant and alternating) studied. The strains of T. pretiosum showed a base temperature $(t b)$ variyng from 8.70 to $13.45^{\circ} \mathrm{C}$, and a thermal constant (K) from 90.09 to 159.43 day degrees.

Key words: Insecta, Trichogrammatidae, biological control, tomato leafminer.

\section{INTRODUÇÃO}

Entre as hortaliças, o tomate é considerado uma das principais culturas devido a sua importância sócio-econômica, pois abrange grandes áreas de produção, condições climáticas favoráveis em quase todo o país, emprego de um grande número de mão-deobra, além da movimentação de um grande volume de

\footnotetext{
${ }^{1}$ Departamento de Fitotecnia, Centro de Ciências Agrárias (CCA), Universidade Federal do Espírito Santo (UFES), 29500-000, Alegre, ES, Brasil.

${ }^{2}$ Departamento de Engenharia Agrícola, CCA, UFES, 29.500-000, Alegre, ES, Brasil.

${ }^{3}$ Departamento de Agronomia/Fitossanidade, Universidade Federal Rural de Pernambuco (UFRPE), 52171-900, Recife, PE, Brasil.

${ }^{4}$ Departamento de Zoologia, Setor de Ciências Biológicas, Universidade Federal do Paraná (UFPR), 81531-990, CP 19.020, Curitiba, PR, Brasil. E-mail: plpastori@yahoo.com.br. Autor para correspondência.
} 
capital em função da comercialização de insumos e produção (MICHEREFF FILHO \& VILELA, 2001).

A traça-do-tomateiro Tuta absoluta (Meyrick, 1917) (Lepidoptera: Gelechiidae) tem sido considerada, desde seu primeiro registro no Brasil, em 1980, uma das principais pragas e tem provocado danos devastadores, com prejuízos expressivos aos agricultores, em todas as regiões produtoras de tomate. Apesar do grande esforço da pesquisa e da difusão tecnológica visando a buscar novas medidas de controle para esta praga, na atualidade o combate ainda é exclusivamente feito através de métodos químicos, com um excessivo número de aplicações de vários ingredientes ativos organo sintéticos, o que tem comprometido a qualidade do produto, a saúde do agricultor, e promovido a contaminação do ambiente (MICHEREFFFILHO \& VILELA, 2001).

As pesquisas têm demonstrado que o manejo integrado é a opção mais viável no controle da traça-do-tomateiro, e o controle biológico, através de parasitóides de ovos com espécies do gênero Trichogramma, a ferramenta mais importante para que se possa manter esta espécie sob níveis populacionais aceitáveis (NAVARRO, 1998, PRATISSOLI \& PARRA; 2001). HAJI et al., (2002) relatam que, em áreas na região do Submédio do São Francisco, onde se implantou um programa de manejo integrado de $\boldsymbol{T}$. absouta $\operatorname{com} \boldsymbol{T}$. pretiosum, ocorreu redução de $55 \%$ nos frutos danificados pela traça-do-tomateiro, em comparação às áreas onde se empregou o controle convencional. Entretanto, o sucesso na introdução ou no desenvolvimento de programas de controle biológico com o uso de Trichogramma depende do pleno conhecimento da potencialidade da espécie e/ou da linhagem a ser empregada e do hospedeiro a ser controlado, além das exigências térmicas e da influência das condições climáticas predominantes (PARRA, 1997; PRATISSOLI \& PARRA, 2000; 2001; PRATISSOLI et al., 2003).

O objetivo desta pesquisa foi estudar a biologia, bem como determinar as exigências térmicas de cinco linhagens de T. pretiosum coletadas em áreas comerciais de tomate, no Estado do Espírito Santo, em ovos da traça-do-tomateiro T. absoluta, sob diferentes temperaturas constantes e flutuantes, visando a fornecer subsídios para o uso destas linhagens no controle da traça-do-tomateiro.

\section{MATERIAL E MÉTODOS}

A pesquisa foi realizada no laboratório de Entomologia do Centro de Ciências Agrárias da Universidade Federal do Espírito Santo (CCA-UFES), sediado na cidade de Alegre-ES. As cinco linhagens de T. pretiosum utilizadas foram provenientes da criação estoque do laboratório e, inicialmente, foram coletadas nas localidades de Rive, a 200m de altitude (L1); Afonso Cláudio, a 380m (L2); Santa Luzia, a 650m (L4); São João, a 750m (L5) e Bela Aurora, a 850m (L6). Para a coleta das linhagens, foram distribuídas, em plantios comerciais de tomate, cartelas de $8 \times 2,5 \mathrm{~cm}$ contendo, em média, 400 ovos de Anagasta kuehniella (Zeller) (Lepidoptera: Pyralidae), que foram coletados após três dias e levados ao laboratório para observação do parasitismo.

Os ovos de $\boldsymbol{T}$. absoluta foram obtidos da criação estoque do laboratório, tendo sido retirados das folhas de tomate com pincel umedecido em água destilada e fixados em retângulos de papel de filtro. Para cada uma das temperaturas, foram utilizadas 30 cartelas contendo 50 ovos com idade inferior a $24 \mathrm{~h}$, isoladas em tubos de vidro de $13,0 \times 1,0 \mathrm{~cm}$, os quais foram fechados com filme plástico de PVC, contendo uma gota de mel puro. Em cada tubo, foram liberadas cinco fêmeas do parasitóide recém-emergidas, permitindo uma exposição ao parasitismo por cinco horas, em câmara climatizada, regulada à $25 \pm 1^{\circ} \mathrm{C}$, umidade relativa de $70 \pm 10 \%$ e fotofase de 14 horas. Ao final deste período, com auxílio de um pincel sob um microscópio estereoscópico, as fêmeas foram retiradas e cada conjunto de tubos foi transferido para câmaras climatizadas, reguladas com umidade relativa de $70 \pm 10 \%$, fotofase de 14 horas e temperaturas constantes, provenientes das estimativas mínimas, médias e máximas $\left(15,20,25\right.$ e $\left.30^{\circ} \mathrm{C}\right)$, e das alternadas, provenientes das estimativas mínimas e máximas anuais $\left(26 / 14\right.$ e $\left.29 / 16^{\circ} \mathrm{C}\right)$ de cada região produtora.

Foram avaliadas a duração do período ovoadulto, realizada através de observações diárias, sempre no mesmo horário; a porcentagem de emergência (viabilidade), efetuada através da contagem dos ovos do hospedeiro que apresentavam orifício de saída dos adultos vistos sob microscópio estereoscópico; e a razão sexual, calculada a partir da fórmula: $r s=n^{\circ} \mathrm{de}$ fêmeas/(no de fêmeas $+\mathrm{n}^{\mathrm{o}}$ de machos), sendo o sexo dos indivíduos determinado com base nas características morfológicas das antenas (BOWEN \& STERN, 1966). O delineamento experimental utilizado foi inteiramente casualizado, com seis tratamentos e 30 repetições com 50 ovos cada. Os resultados foram submetidos à análise de variância e as médias comparadas pelo teste de Tukey a 5\% de probabilidade de erro.

A partir dos resultados obtidos para a velocidade de desenvolvimento, nas temperaturas constantes $\left(15,20,25\right.$ e $\left.30^{\circ} \mathrm{C}\right)$, procedeu-se a 
determinação das exigências térmicas das linhagens de T. pretiosum, através do calculo do limiar térmico inferior (tb) e da constante térmica (K). Para isso, utilizou-se o método da hipérbole (HADDAD et al., 1995), através do programa estatístico "MOBAE", desenvolvido pelo Departamento de Entomologia ESALQ/USP.

\section{RESULTADOS E DISCUSSÃO}

A duração do ciclo de desenvolvimento das cinco linhagens (L1, L2, L4, L5 e L6) de T. pretiosum sobre ovos da traça-do-tomateiro T. absoluta foi afetada significativamente pela temperatura, ocorrendo aumento na velocidade de desenvolvimento com a elevação térmica na faixa de temperatura constante (15 a $30^{\circ} \mathrm{C}$ ). A maior duração do ciclo foi observada na temperatura de $15^{\circ} \mathrm{C}$ e a menor a $30^{\circ} \mathrm{C}$. Nas temperaturas alternadas $\left(26 / 14\right.$ e $\left.29 / 16^{\circ} \mathrm{C}\right)$, independentemente da linhagem, o período do ciclo de desenvolvimento foi estatisticamente semelhante ao obtido na faixa de temperatura de 20 a $30^{\circ} \mathrm{C}$ (Tabela 1 ).

A duração do ciclo de todas as linhagens, nas temperaturas de $15,25,30^{\circ} \mathrm{Ce}$ na alternada $29 / 16^{\circ} \mathrm{C}$, não diferiu (Tabela 1). A $20^{\circ} \mathrm{C}$, com exceção da linhagem L2, para todas as demais, os valores foram estatisticamente semelhantes. Já na temperatura alternada de $26 / 14^{\circ} \mathrm{C}$, as linhagens L4 e L5 apresentaram as menores taxas de duração do ciclo, diferindo significativamente das demais.

Dentre os fatores físicos, a temperatura é aquele que tem sido citado como o de maior influência sobre os parâmetros biológicos de espécies de Trichogramma, tais como: duração do ciclo de desenvolvimento, fecundidade, razão sexual, viabilidade e longevidade (BLEICHER \& PARRA, 1989; GRILLÉ \& BASSO, 1994; PRATISSOLI \& PARRA, 2000).
Foi observado um aumento na velocidade de desenvolvimento em função da elevação térmica (Figura 1), dentro da faixa de temperatura constante, para todas as linhagens estudadas, sendo a influência da temperatura significativa (Tabela 1). Desta mesma forma, PRATISSOLI \& PARRA(2000), trabalhando com T. pretiosum sobre ovos de T. absoluta, verificaram o mesmo aumento significativo da velocidade de desenvolvimento em função da elevação térmica que o observado para as cinco linhagens aqui estudadas.

De um modo geral, as linhagens de $\boldsymbol{T}$. pretiosum avaliadas, dentro de cada temperatura, não apresentaram diferenças discrepantes para a duração do ciclo de desenvolvimento. Fato este também verificado em relação a outros trabalhos, porém dentro da faixa de temperatura constante de 20 a $30^{\circ} \mathrm{C}$ (CALVIN et al., 1984; HARRISON et al., 1985; PRATISSOLI \& PARRA, 2000). No entanto, na temperatura de $15^{\circ} \mathrm{C}$, todas as linhagens apresentaram um ciclo de desenvolvimento longo, sendo duas vezes e meia superior ao encontrado a $20^{\circ} \mathrm{C}$, o que poderá comprometer um programa de controle biológico nessas condições de temperatura. No trabalho de PRATISSOLI \& PARRA (2000), à temperatura de $20^{\circ} \mathrm{C}$, verificou-se que a duração do ciclo foi maior, em média, 3,8 dias, que os obtidos neste trabalho.

Os resultados obtidos neste estudo mostram que a viabilidade das cinco linhagens de $\boldsymbol{T}$. pretiosum criadas em ovos de T. absoluta foi afetada pela variação da temperatura (Tabela 2). As melhores taxas de emergência foram verificadas, para a $\mathrm{L} 1$, nas temperaturas de 30 e $26 / 14^{\circ} \mathrm{C}$; para $\mathrm{L} 2$, a $25^{\circ} \mathrm{C}$ e nas temperaturas alternadas; para $\mathrm{L} 4 \mathrm{e} \mathrm{L6}$, a $29 / 16^{\circ} \mathrm{C}$, e para $\mathrm{L} 5$, nas temperaturas de 25,30 e $26 / 14^{\circ} \mathrm{C}$. As menores taxas de emergência para todas as linhagens estudadas foram constatadas na temperatura de $15^{\circ} \mathrm{C}$.

Tabela 1 - Duração (dias) (Média \pm EP) do ciclo de desenvolvimento (ovo-adulto) de cinco linhagens de Trichogramma pretiosum criadas em ovos de Tuta absoluta, em temperaturas (T) constantes e flutuantes. $70 \pm 10 \%$ de umidade relativa e fotofase de 14 horas. Laboratório de Entomologia, CCA-UFES ( $\mathrm{n}=30)$.

\begin{tabular}{lccccc}
\hline & \multicolumn{3}{c}{ Linhagem de $\boldsymbol{T}$. pretiosum } \\
\cline { 2 - 6 }$\left({ }^{\circ} \mathrm{C}\right)$ & $\mathrm{L} 1$ & $\mathrm{~L} 2$ & $\mathrm{~L} 4$ & $\mathrm{~L} 5$ & $\mathrm{~L} 6$ \\
\hline 15 & $35,0 \pm 0,07 \mathrm{Aa}^{*}$ & $36,8 \pm 0,12 \mathrm{Aa}$ & $35,8 \pm 0,08 \mathrm{Aa}$ & $32,4 \pm 0,17 \mathrm{Aa}$ & $36,7 \pm 0,07 \mathrm{Aa}$ \\
20 & $14,2 \pm 0,09 \mathrm{Ba}$ & $10,2 \pm 0,05 \mathrm{Bb}$ & $15,5 \pm 0,20 \mathrm{Ba}$ & $15,2 \pm 0,05 \mathrm{Ba}$ & $14,9 \pm 0,13 \mathrm{Ba}$ \\
25 & $9,5 \pm 0,18 \mathrm{BCa}$ & $11,8 \pm 0,10 \mathrm{Ba}$ & $10,9 \pm 0,36 \mathrm{Ca}$ & $12,7 \pm 0,08 \mathrm{Ca}$ & $11,0 \pm 0,14 \mathrm{Ca}$ \\
30 & $6,7 \pm 0,13 \mathrm{Ca}$ & $7,4 \pm 0,05 \mathrm{Ca}$ & $5,2 \pm 0,08 \mathrm{Da}$ & $6,9 \pm 0,04 \mathrm{Da}$ & $8,0 \pm 0,03 \mathrm{Da}$ \\
$26 / 14$ & $14,8 \pm 0,10 \mathrm{Ba}$ & $13,5 \pm 0,10 \mathrm{Ba}$ & $10,2 \pm 0,01 \mathrm{Cb}$ & $8,0 \pm 0,05 \mathrm{Db}$ & $13,7 \pm 0,06 \mathrm{Ca}$ \\
$29 / 16$ & $12,4 \pm 0,12 \mathrm{Ba}$ & $12,8 \pm 0,18 \mathrm{Ba}$ & $11,4 \pm 0,20 \mathrm{Ca}$ & $9,9 \pm 0,04 \mathrm{CDa}$ \\
\hline
\end{tabular}

*Médias não seguidas de mesma letra, maiúscula na coluna e minúscula na linha, diferem estatisticamente entre si em nível de 5\% de probabilidade de erro pelo Teste de Tukey. 

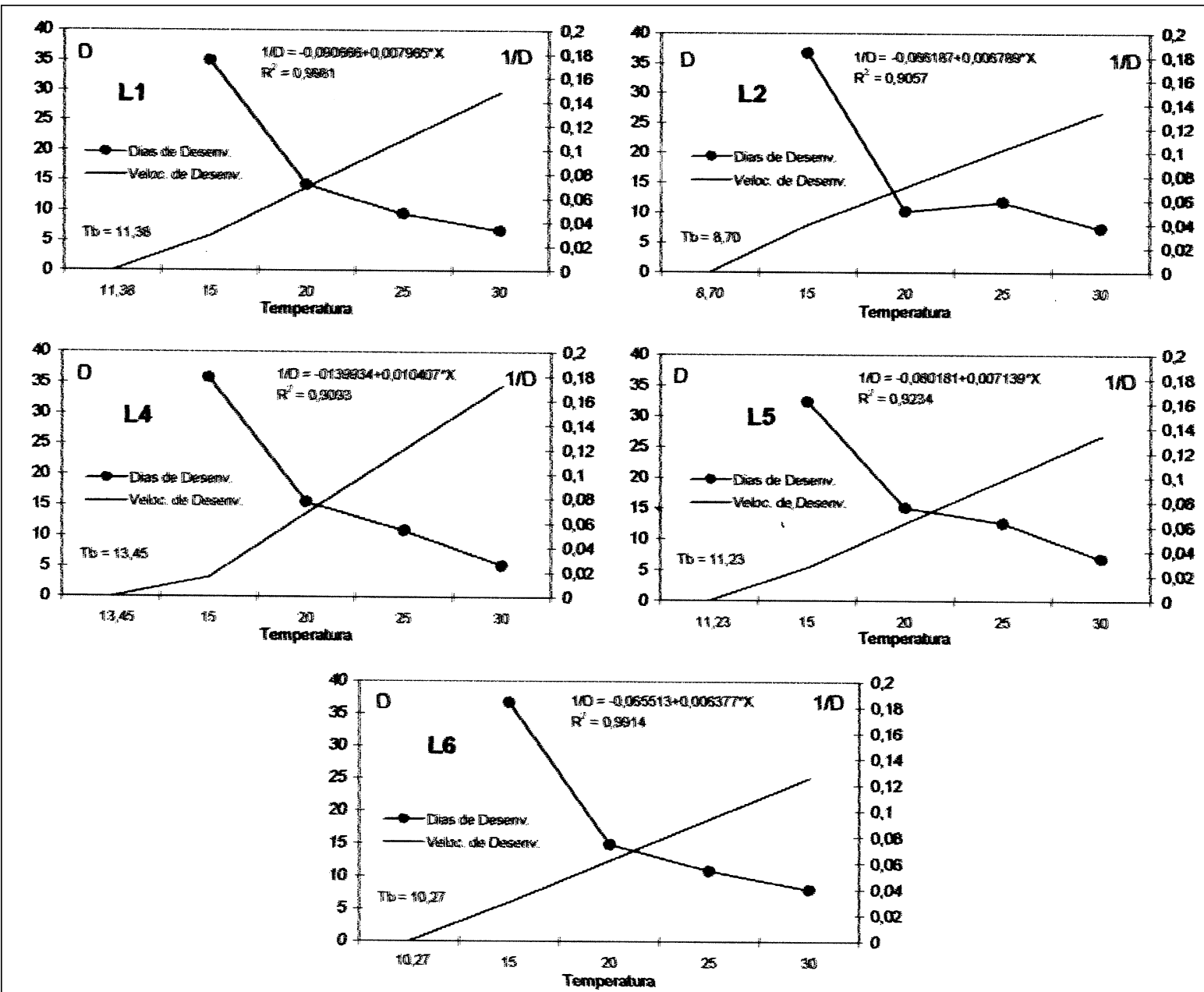

Figura 1 - Curva de velocidade de desenvolvimento de cinco linhagens de Trichogramma pretiosum em ovos de Tuta absoluta, em diferentes temperaturas. $70 \pm 10 \%$ de umidade relativa e fotofase de 14 horas. Laboratório de Entomologia, CCA-UFES.

Ao se comparar à viabilidade das linhagens, dentro de cada temperatura (Tabela 2), verificou-se uma grande variabilidade comportamental. Ao se avaliar o desempenho das linhagens nas diferentes temperaturas, constatou-se que as linhagens L2 e L5 apresentaram uma concentração dos melhores resultados quanto à taxa de emergência (Tabela 2). Esses resultados, quando confrontados com os de outros trabalhos, apresentaram comportamento semelhante (PARRA et al., 1991; PARRA \& SALES Jr., 1994; PRATISSOLI \& PARRA, 2000). No entanto, resultados divergentes podem ser encontrados, como os obtidos por BLEICHER \& PARRA (1989), que estudaram três populações de $\boldsymbol{T}$. pretiosum e não encontraram variação na viabilidade das mesmas, mesmo em temperaturas extremas.

Independentemente das temperaturas estudadas, as maiores porcentagens de emergência foram obtidas em temperaturas superiores a $20^{\circ} \mathrm{C}$ para todas as linhagens (Tabela 2). No entanto, não foi observado viabilidade superior a $80 \%$. Nos estudos de bioecologia elaborados para os parasitóides de ovos do gênero Trichogramma, a faixa de temperatura de 22 a $30^{\circ} \mathrm{C}$ tem-se mostrado como aquela em que são obtidas as melhores taxas de emergência (HARRISON et al., 1985; PRATISSOLI \& PARRA, 2000). Em temperaturas inferiores a $20^{\circ} \mathrm{C}$, diversas pesquisas têm demonstrado que a porcentagem de emergência é reduzida(CONSÔLI \& PARRA, 1991; PARRA\& SALES Jr., 1994; PRATISSOLI \& PARRA, 2000). Esses resultados também foram observados neste estudo, porém com valores inferiores. Os baixos valores da viabilidade, encontrados à temperatura de $15^{\circ} \mathrm{C}$, podem estar relacionados ao fato de esta ser muita próxima à temperatura base dessas linhagens (Figura 1), o que a torna inadequada para a criação desse parasitóide. A viabilidade das cinco linhagens foi inferior a $50 \%$ nas temperaturas 15 e $20^{\circ} \mathrm{C}$ e não atingiu $80 \%$ nas demais 
Tabela 2 - Viabilidade (Média \pm EP) de cinco linhagens de Trichogramma pretiosum criadas em ovos de Tuta absoluta, em temperaturas constantes e flutuantes. $70 \pm 10 \%$ de umidade relativa e fotofase de 14 horas. Laboratório de Entomologia, CCA-UFES (n=30).

\begin{tabular}{|c|c|c|c|c|c|}
\hline \multirow{2}{*}{$\mathrm{T}\left({ }^{\circ} \mathrm{C}\right)$} & \multicolumn{5}{|c|}{ Linhagem de $T$. pretiosum } \\
\hline & L1 & $\mathrm{L} 2$ & $\mathrm{~L} 4$ & L5 & L6 \\
\hline 15 & $0,8 \pm 0,37 \mathrm{~Eb}^{*}$ & $1,8 \pm 1,00 \mathrm{Db}$ & $5,6 \pm 1,27 \mathrm{Fa}$ & $6,3 \pm 1,28 \mathrm{Ca}$ & $6,2 \pm 1,65 \mathrm{Ea}$ \\
\hline 20 & $15,1 \pm 2,71 \mathrm{Dc}$ & $9,7 \pm 2,16 \mathrm{Cd}$ & $36,5 \pm 3,17 \mathrm{~Eb}$ & $41,0 \pm 3,03 \mathrm{Ba}$ & $40,4 \pm 2,70 \mathrm{Da}$ \\
\hline 25 & $50,1 \pm 4,66 \mathrm{Bc}$ & $62,7 \pm 3,58 \mathrm{Aa}$ & $63,9 \pm 2,41 \mathrm{Ba}$ & $58,6 \pm 2,27 \mathrm{Ab}$ & $66,4 \pm 3,25 \mathrm{Ba}$ \\
\hline 30 & $59,5 \pm 4,56 \mathrm{Aa}$ & $53,3 \pm 2,43 \mathrm{Bb}$ & $40,6 \pm 1,20 \mathrm{Dc}$ & $59,7 \pm 4,78 \mathrm{Aa}$ & $52,8 \pm 2,62 \mathrm{Cb}$ \\
\hline $26 / 14$ & $62,8 \pm 5,66 \mathrm{Aa}$ & $60,8 \pm 2,47 \mathrm{Aa}$ & $58,7 \pm 5,46 \mathrm{Cb}$ & $62,0 \pm 5,66 \mathrm{Aa}$ & $38,2 \pm 5,25 \mathrm{Dc}$ \\
\hline $29 / 16$ & $44,1 \pm 2,66 \mathrm{Cc}$ & $64,9 \pm 3,74 \mathrm{Ab}$ & $79,2 \pm 5,50 \mathrm{Aa}$ & $40,0 \pm 3,08 \mathrm{Bc}$ & $73,8 \pm 4,24 \mathrm{Aa}$ \\
\hline
\end{tabular}

*Médias não seguidas de mesma letra, maiúscula na coluna e minúscula na linha, diferem estatisticamente entre si em nível de 5\% de probabilidade de erro pelo Teste de Tukey.

temperaturas estudadas. Estes baixos valores de viabilidade podem estar relacionados à possível degeneração das linhagens pela criação consecutiva em laboratório e sobre hospedeiro alternativo. Este fato tem sido relatado, visto que, dentre as técnicas de produção de Trichogramma, pode ocorrer a deterioração das características naturais desse parasitóide e, com isso, não se conseguir preservar a estrutura genética da população (HASSAN, 1993).

A razão sexual das cinco linhagens de Trichogramma foi afetada pela temperatura (Tabela 3 ), havendo diferença entre os valores obtidos nas faixas das temperaturas estudadas. A temperatura tem sido relacionada por diversos autores como o fator físico que pode interferir na razão sexual desses parasitóides de ovos (PARRA \& SALES Jr., 1994; PRATISSOLI \& PARRA, 2000). No entanto, outros pesquisadores afirmam que a condição térmica não afeta essa relação sexual (CALVIN et al., 1984; BLEICHER \& PARRA, 1989). No presente estudo, verificou-se que apenas a linhagem L1, nas temperaturas alternadas, atingiu uma razão sexual superior a 0,5 , sendo que, para as demais linhagens, e nas temperaturas estudadas (constantes e alternadas), esse índice foi bem inferior. Isso indica que, na relação, o número de machos foi extremamente superior ao de fêmeas, o que é indesejável em programas de controle biológico, pois compromete a eficiência desses parasitóides de ovos a nível de campo. Este fato pode estar relacionado à aceitação do hospedeiro T. absoluta pelas linhagens de $T$. pretiosum estudadas, bem como à possível degeneração das últimas, quando criadas em laboratório, visto que PRATISSOLI \& PARRA (2000), trabalhando com este parasitóide sobre o mesmo hospedeiro utilizado neste trabalho, observaram uma razão sexual superior a 0,95 , em temperaturas compreendidas na faixa de 18 a $32^{\circ} \mathrm{C}$.

Para a linhagem L1 de T. pretiosum, quando criada em ovos de T. absoluta, os valores do limite térmico inferior de desenvolvimento (tb) e a constante térmica $(\mathrm{K})$ foram de $11,38^{\circ} \mathrm{C}$ e 125,62 graus-dias respectivamente; para a $\mathrm{L} 2$, esses valores foram de $8,70^{\circ} \mathrm{C}$ e 154,43 graus-dia; para $\mathrm{L} 4$, de $13,45^{\circ} \mathrm{C}$ e 96,09 graus-dia; L5 de $11,23^{\circ} \mathrm{C}$ e 140,07 graus-dia e para L6 de $10,27^{\circ} \mathrm{Ce} 157,32$ graus-dia (Tabela 4$)$.

Tabela 3 - Razão sexual (Média \pm EP) de cinco linhagens de Trichogramma pretiosum criadas em ovos de Tuta absoluta, em temperaturas constantes e flutuantes. $70 \pm 10 \%$ de umidade relativa e fotofase de 14 horas. Laboratório de Entomologia, CCA-UFES (n=30).

\begin{tabular}{|c|c|c|c|c|c|}
\hline \multirow{2}{*}{$\mathrm{T}\left({ }^{\circ} \mathrm{C}\right)$} & \multicolumn{5}{|c|}{ Linhagem de $\boldsymbol{T}$. pretiosum } \\
\hline & $\mathrm{L} 1$ & L2 & $\mathrm{L} 4$ & L5 & L6 \\
\hline 15 & $0,00 \pm 0,00 \mathrm{Dc}^{*}$ & $0,00 \pm 0,00 \mathrm{Cc}$ & $0,16 \pm 0,02 \mathrm{Bb}$ & $0,28 \pm 0,01 \mathrm{Aa}$ & $0,18 \pm 0,04 \mathrm{Cb}$ \\
\hline 20 & $0,12 \pm 0,04 \mathrm{Cc}$ & $0,22 \pm 0,02 \mathrm{Ab}$ & $0,15 \pm 0,01 \mathrm{Bc}$ & $0,22 \pm 0,01 \mathrm{Bb}$ & $0,37 \pm 0,04 \mathrm{Aa}$ \\
\hline 25 & $0,18 \pm 0,03 \mathrm{Ca}$ & $0,11 \pm 0,01 \mathrm{Bb}$ & $0,16 \pm 0,02 \mathrm{Ba}$ & $0,18 \pm 0,02 \mathrm{Ca}$ & $0,11 \pm 0,03 \mathrm{CDb}$ \\
\hline 30 & $0,25 \pm 0,05 \mathrm{Bbc}$ & $0,21 \pm 0,01 \mathrm{Ac}$ & $0,37 \pm 0,02 \mathrm{Aa}$ & $0,19 \pm 0,01 \mathrm{Cc}$ & $0,30 \pm 0,02 \mathrm{Bb}$ \\
\hline $26 / 14$ & $0,52 \pm 0,05 \mathrm{Aa}$ & $0,11 \pm 0,01 \mathrm{Bc}$ & $0,09 \pm 0,04 \mathrm{Cc}$ & $0,18 \pm 0,02 \mathrm{Cb}$ & $0,12 \pm 0,02 \mathrm{CDc}$ \\
\hline $29 / 16$ & $0,57 \pm 0,03 \mathrm{Aa}$ & $0,14 \pm 0,03 \mathrm{Bc}$ & $0,05 \pm 0,02 \mathrm{Cd}$ & $0,23 \pm 0,01 \mathrm{Bb}$ & $0,08 \pm 0,02 \mathrm{Dd}$ \\
\hline
\end{tabular}

*Médias não seguidas de mesma letra, maiúscula na coluna e minúscula na linha, diferem estatisticamente entre si em nível de 5\% de probabilidade de erro pelo Teste de Tukey. 
Tabela 4 - Temperatura base (tb), constante térmica (K) e coeficiente de determinação $\left(\mathrm{R}^{2}\right)$ de cinco linhagens de Trichogramma pretiosum criadas em ovos de Tuta absoluta, em temperaturas constantes. $70 \pm$ $10 \%$ de umidade relativa e fotofase de 14 horas. Laboratório de Entomologia, CCA-UFES ( $n=30)$.

\begin{tabular}{clll}
\hline Linhagem de $\boldsymbol{T}$. pretiosum & $\mathrm{Tb}\left({ }^{\circ} \mathrm{C}\right)$ & $\mathrm{K}(\mathrm{GD})$ & $\mathrm{R}^{2}(\%)$ \\
\hline L1 & 11,38 & 125,62 & 99,81 \\
L2 & 8,70 & 154,43 & 90,57 \\
L4 & 13,45 & 96,09 & 90,93 \\
L5 & 11,23 & 140,07 & 92,34 \\
L6 & 10,27 & 157,32 & 99,14 \\
\hline
\end{tabular}

Analisando os resultados de tb e $\mathrm{K}$, presentes na tabela 4 , quando comparados com os reportados por CALVIN et al. (1984), de $9,97^{\circ} \mathrm{C}$ e 158,71 graus-dia; por BLEICHER \& PARRA(1990), de $12,81^{\circ} \mathrm{C}$ e 133,25 graus-dia e por PRATISSOLI \& PARRA, (2000), de $13,53^{\circ} \mathrm{C}$ e 120,90 graus-dia para diferentes linhagens T. pretiosum, em ovos de Diatraea grandiosella Dyar (Lepidoptera: Crambidae), A. kuehniela e Phthorimaea operculella (Zeller) (Lepidoptera: Gelechiidae), respectivamente, constatou-se que a temperatura base obtida para a linhagem L2 foi ligeiramente inferior, sendo que, para as demais, os valores foram próximos. Através desta mesma comparação, pode-se constatar que as variações ocorridas nos valores de tb e $\mathrm{K}$ foram em função da linhagem de $\boldsymbol{T}$. pretiosum e do hospedeiro de criação. Apenas a linhagem L4 do presente estudo apresentou temperatura base superior aos resultados obtidos por PRATISSOLI \& PARRA (2000), que, trabalhando com $\boldsymbol{T}$. pretiosum sobre ovos de $\boldsymbol{T}$. absoluta, obtiveram temperatura base de $12,98^{\circ} \mathrm{C}$ e constante térmica de 131,33 graus-dia. Essas variações de tb e K também foram verificadas em outras espécies de Trichogramma (BLEICHER \& PARRA, 1990; PARRA et al., 1991).

\section{CONCLUSÕES}

A temperatura interfere nas características biológicas das linhagens de $\boldsymbol{T}$. pretiosum estudadas. A velocidade de desenvolvimento aumenta com a elevação térmica. As variações ocorridas na temperatura base e na constante térmica foram em função das linhagens de $\boldsymbol{T}$. pretiosum.

\section{AGRADECIMENTOS}

Ao Conselho Nacional de Desenvolvimento Científico e Tecnológico $(\mathrm{CNPq})$ e à Financiadora de Estudos e Projetos (FINEp), pelas bolsas e auxílios concedidos.

\section{REFERÊNCIAS}

BLEICHER, E.; PARRA, J.R.P. Espécies de Trichogramma parasitóides de Alabama argillacea. I. Biologia de três populações. Pesquisa Agropecuária Brasileira, v.24, n.8, p.929-940, 1989.

BLEICHER, E.; PARRA, J.R.P. Espécies de Trichogramma parasitóides de Alabama argillacea. III. Determinação das exigências térmicas de três populações. Pesquisa Agropecuária Brasileira, v.25, n.2, p.215-219, 1990.

BOWEN, W.R.; STERN, V.M. Effect of temperature on the production of males and sexual mosaics in a uniparental race of Trichogramma semifunatum (Hymenoptera: Trichogrammatidae). Entomological Society of America Annals, v.59, n.4, p.823-834, 1966.

CALVIN, D.D. et al. Impact of environmental factors on Trichogramma pretiosum reared on Southwestern corn borer eggs. Environmental Entomology, v.13, n.3, p.774-780, 1984 .

CÔNSOLI, F.L.; PARRA, J.R.P. Effect of constant and alternating temperatures on Trichogramma galloi Zucchi biology. I. Developmental and thermal requirements. Journal of Applied Entomology, v.119, p.415-418, 1991.

GRILLÉ, G.; BASSO, C. Biology, thermal requirements and performance of Trichogramma pretiosum Riley and T. galloi Zucchi under laboratory conditions. Les Colloques de I' INRA, v.73, p.79-82, 1994.

HADDAD, M.I. et al. Modelos estatísticos aplicados à entomologia. Piracicaba: FEALQ, 1995. 44p.

HAJI, F.N.P. et al. Trichogramma pretiosum para o controle de pragas no tomateiro industrial. In: PARRA, J.R.P. et al. Controle biológico: parasitóides e predadores. São Paulo: Manole, 2002. Cap.28, p.477-493.

HARRISON, W.W. et al. Development of Trichogramma exiguum and T. pretiosum at five temperature regimes. Environmental Entomology, v.14, n.2, p.118-121, 1985.

HASSAN, S.A. The mass rearing and utilization of Trichogramma to control lepidopterous pests: Achievements and outlook. Pesticides Science, v.37, p.387-391, 1993.

MICHEREFF FILHO, M.; VILELA, E.F. Traça do tomateiro, Tuta absoluta (Lep.: Gelechiidae). In: VILELA, E.A. et al. Pragas introduzidas no Brasil. Ribeirão Preto: Holos, 2001. Cap.11, p.81-84.

NAVARRO, M.A. Trichogramma spp. Produción, uso y manejo en Colombia. Valle del Cauca: Guadalajara de Buga, 1998. 176p.

PARRA, J.R.P. Técnicas de criação de Anagasta kuehniella, hospedeiro alternativo para produção de Trichogramma. In: PARRA, J.R.P.; ZUCCHI, R.A. Trichogramma e o controle biológico aplicado. Piracicaba: FEALQ, 1997. Cap.4, p.121150 .

PARRA, J.R.P. et al. Biology, thermal requirements of Trichogramma galloi Zucchi and T. distinctum Zucchi, on two 
alternative hosts. Les Colloques de I' INRA, v.56, p.81-84, 1991.

PARRA, J.R.P.; SALES JR., O. Biology of Trichogramma galloi Zucchi reared on natural host and factitions hosts under diferents temperatures and relatives humidities. Les Colloques de I' INRA, v.73, p.95-100, 1994.

PRATISSOLI, D.; PARRA, J.R.P. Desenvolvimento e exigências térmicas de Trichogramma pretiosum Riley criado em duas traças do tomateiro. Pesquisa Agropecuária Brasileira, v.35, n.7, p.1281-1288, 2000.
PRATISSOLI, D.; PARRA, J.R.P. Seleção de linhagens de Trichogramma pretiosum Riley (Hym.: Trichogrammatidae) para o controle das traças do tomateiro Tuta absoluta (Meyrick) e Phthorimaea operculella (Zeller) (Lepidoptera: Gelechiidae). Neotropical Entomology, v.30, n.2, p.277-282, 2001.

PRATISSOLI, D. et al. Ocorrência de Trichogramma pretiosum em áreas comerciais de tomate, no Espírito Santo, em regiões de diferentes altitudes. Horticultura Brasileira, v.21, n.1, p.73-76, 2003. 